\title{
An Image Reconstruction Model under Poisson Noise Using multiscale Compressed Sensing
}

\author{
Pai Zhang \\ College of Intelligence and Information Engineering \\ Tangshan College, China
}

Received: August 14, 2020. Revised: September 5, 2020. Accepted: September 21, 2020. Published: September 24, 2020

\begin{abstract}
For the influence of poisson noise images, in order to get rid of poisson noise, this paper put forward image reconstruction method by using multiscale compressed sensing. the algorithm can approximate the optimal sparse representation of the image edge details such as the characteristics of theShearlet domain based multi-scale compressed sensing method. The image is decomposed into the high-frequency subbands byShearlet, and the compressed sensing is applied into each subband to reconstruct the image. In this paper, A total variation of RL iterative algorithm constructed by nonlinear projection algorithm based on closed convex set is explored as the reconstruction method, which use derivation of the nonlinear projection instead of total variation. In mathematics, Shearlet has been proved to be a better tool for edge characterization than traditional wavelet. By using the nonlinear projection scheme to constrain the residual coefficients in the Shearlet domain, a better estimation can be obtained from the Shearlet representation. Numerical examples show that the denoising effect of these methods is very good, which is better than the correlation method based on Curvelet transform. In addition, the number of iterations required by our scheme is far less than that of our competitors.
\end{abstract}

Keywords-Image reconstruction,Poisson noise,nonlinear projection, image reconstruction.

\section{INTRODUCTION}

$\mathrm{B}$ iomedical and astronomical imaging systems are usually disturbed by Poisson noise[1,2]. In order to solve the deconvolution problem of Poisson noise, scholars have proposed some models and algorithms, such as Tikhonov Miller filter and Richardson Lucy (RL) [3] algorithm. The RL algorithm based on maximum likelihood (ML) estimation can adapt to the statistical characteristics of Poisson noise, It has been widely used, but after many iterations, the algorithm will amplify the noise, which is more serious in the case of low signal-to-noise ratio. This problem can be solved by introducing the regularization term. Based on this, the RL algorithm of total variation (TV) regularization is proposed in reference [4], Many authors use RL algorithm of wavelet regularization. Bayesian and multi-scale analysis framework, literature [5] gives another method, which decomposes Poisson likelihood function in multi-scale, and constrains the regularity of solution by prior probability distribution, and further applies expectation maximum (EM) [6]algorithm to solve the maximum posterior probability. However, this algorithm is only applicable to Haar multi-scale analysis based on wavelet.

Compressed sensing (CS) is a new data sampling theory proposed by Donoho et al[7]. It considers that if a signal is compressible or sparse in a certain transform domain, the signal can be projected onto a low dimensional space by using a transform independent observation matrix.Then, the reconstructed image can be obtained by using the optimization method. It can be proved that such projection contains the reconstructed signal Enough information. Multiscale compressed sensing $[8,9]$ is an extension of traditional compressed sensing, which is based on the known signal. After sparse decomposition of a signal, low-frequency and a series of high-frequency word bands can be obtained. MCS only reconstructs the high-frequency word band. Because the low-frequency information contains most of the energy, it retains all the information. When the signal itself or in the representation of a certain basis function is sparse enough, the quality of the reconstructed signal with multi-scale compressed sensing is significantly better than that of the traditional CS with the same number of observations. Compared with the traditional wavelet transform, the beyond wavelet transform can more sparsely represent the image edge, contour and other information, so the effect of image reconstruction is more ideal[10-12]. Since discreteShearlet transform (DST) [13]can approximate the optimal sparse representation of images, our work proposes a multiscale compressed sensing, which decomposes image inShearlet domain(MCS), After the image is decomposed by DST, all the low-frequency information is retained, and the directional sub-band information of each scale in the high-frequency region is observed and reconstructed by CS respectively. MCS is applied to the image with Poisson noise to restore the image. At the reconstruction end, a RL algorithm based on nonlinear projection for the total difference regularization term is proposed. The nonlinear projection on a closed convex set $[14,15]$ can be used to replace the derivation of the regular term, Thus, more texture details can be retained. The experimental results also show that the proposed algorithm is better than RL-TV algorithm in reconstruction effect.

\section{THE BASIC THEORY OF MCS METHOD}

The theoretical basis ofShearlet transform is synthetic wavelet. The decomposition ofShearlet transform consists of the following two steps: multi-scale subdivision and directional localization.

(1) Multiscale subdivision. Haar wavelet is used to decompose the image. For example, the low-frequency coefficients and the high-frequency coefficients at different scales are obtained,(is the scale of decomposition).

(2) Direction localization. In order to obtain the high frequency components in different directions, The high frequency coefficients at scale are divided by window functions with direction and scale variation. On the high 
frequency subbands, $\mathrm{D}_{0}$ and $\mathrm{D}_{1}$ regions are decomposed by $2^{j+1}+1$ window function.

According to the traditional CS theory, if $x \in R^{N}$, which represents the known signal,are approximately represented by $K(K<N)$ non-zero values under a certain basis function, then the definition is sparse in the domain. A measurement matrix $\Phi \in R^{m \times N} \quad(m<<N)$ is constructed to observe the signa $y=\Phi x \quad\left(y \in R^{m}\right)$, and the measured value $y$ can be considered to contain all important information of original $\mathrm{x}$ when measurement times $m>(C \cdot K) \cdot \log (N)$. The process of when measurement times $m>(C \cdot K) \cdot \log (N)$. The process of observation signal adopted here is non adaptive, and the measurement matrix $\Phi$ does not depend on the structure of the signal. By solving the optimization problem under $\ell_{0}$ norm[16], the signal can be reconstructed accurately, that is $\min \left\|\Psi^{T} \hat{x}\right\|_{0}$ s.t. $y=\Phi \Psi^{T} x$. Since it is a NP-hard problem for the solution of optimization problem under norm, the solution method is often transformed into the solution of optimization problem under $\ell_{1}$ norm with equivalent relation[17],that is
The key problem of CS theory is signal sparsity. For most natural images, they are non sparse, the quality of reconstructed image can be significantly improved by applying CS directly to the sparse part of the signal in the transform domain after effectively sparse representation by using effective image transformation. Among the existing multiscale image transforms, Guo et al. Constructed the discreteShearlet transform.Is constructed based on the theory of composite wavelet. The affine system is used to associate geometry and multi-scale analysis, which can realize the "thinnest" representation of image edge. The most important feature of discreteShearlet transform (DST) is to use Laplacian pyramid (LP) defined in frequency domain of pseudo polar lattice to cascade frequency domain directional filter banks (FDFB) to realize multi-scale and multi-directional decomposition of two-dimensional image. Because the spectrum of each directional filter in FDFBsatisfies the tight support, the directional filter does not produce spectrum aliasing. In Fig.1,it is presented an example of image spectrum segmentation without leakage using six directional filters in pseudo polar array frequency domain.

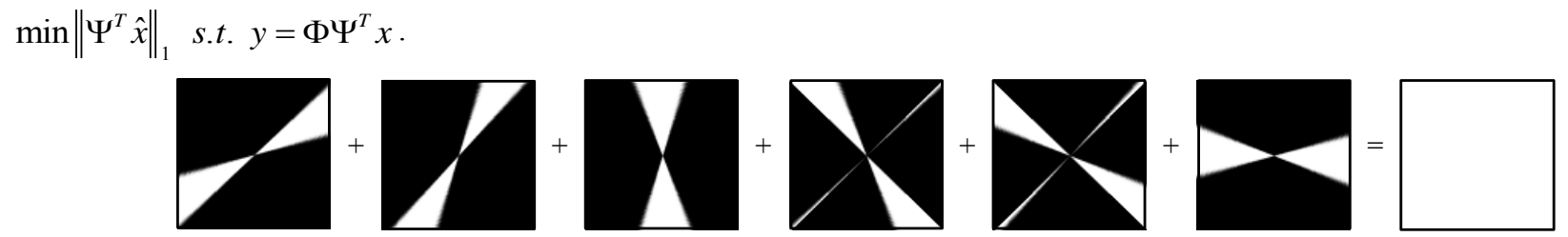

Fig.1. In PPFC, FDFB(with six directional filters) splits the spectrum without leakage

The original image $f$ which decomposed by DST, is expressed as follows:

$$
f=\alpha_{p_{0}} \phi_{p_{0}}+\sum_{p=p_{0}}^{P} \sum_{d} \theta_{p, s} \varphi_{p, s}
$$

$\phi_{p_{0}}, \varphi_{p, s}$ are the scale and Shearlet function respectively. Since $\alpha_{p_{0}}$ contains most of the energy of the image, it is almost not sparse, so all the information of $\alpha_{p_{0}}$ is retained. The directional subband information $\theta_{p, s}$ of high frequency scale, which is the edge, texture and other details of the image, has strong sparsity. By using this property, the CS is only applied into o $\theta_{p, s}$

$$
y_{p, s}=\Phi_{p, s} \alpha_{p, s}
$$

By solving the optimization problem, it is obtained $\hat{\theta}_{p, s}$

$$
\min \left\|\hat{\theta}_{p, s}\right\|_{T V} \text { s.t. } \quad y_{p, s}=\Phi_{p, s} \alpha_{p, s}
$$

Finally, the image reconstructed by MCS method is as $Y=\alpha_{p_{0}}^{Y} \varphi_{p_{0}}+\sum_{p=p_{0}}^{P} \sum_{s} \hat{\theta}_{p, s}^{Y} \phi_{p, s}$. The MCS method can reconstruct the image accurately with less sampling points, which is superior to the traditional CS algorithm

\section{IMPROVED RL TV ALGORITHM}

\section{A. Construction of image deconvolution Chambolle projection}

In image processing, the labeling model of deconvolution of image restoration $[18,19]$ is as follows:

$$
\min _{u \in X} \frac{\|R u-g\|^{2}}{2 \lambda}+J(u)
$$

Where: $\mathrm{x}$ is the two-dimensional image space, $\mathrm{u}$ is the original image to be restored, GX is the observed degraded image, $\mathrm{R}$ is the degenerate convolution operator, $\lambda>0$ is a constant and $|\mathrm{u}|^{2}=<u, u>$ represents the inner product.

Let $\mathrm{v}=R u$, suppose $\mathrm{R}$ is reversible, then the above formula can be transformed into:

$$
\min _{v \in X} \frac{\|v-g\|^{2}}{2 \lambda}+J\left(R^{-1} v\right)
$$


If the optimal solution of the above formula is solved, the optimal solution of the original image $u$ to be restored will also be solved.

The Euler equation of the above formula is as follows:

$$
\frac{1}{\lambda}(v-g)+\left(R^{-1}\right)^{\top} \partial J\left(R^{-1} v\right) \ni 0
$$

The results are as follows: $\frac{R^{T}(\mathrm{~g}-\mathrm{v})}{\lambda} \in \partial J\left(R^{-1} v\right)$, The dual property of Legendre is obtained as: $R^{-1} v \in \partial J^{*}\left(\frac{R^{T}(g-v)}{\lambda}\right)$ let $\hat{w}=\frac{R^{T}}{\lambda}(\mathrm{g}-\mathrm{v}), w$ is the optimal solution of the formula below

$$
\min _{w}=\frac{1}{2}\left\|\mathrm{w}-\frac{R T g}{\lambda}\right\|^{2}+\frac{R^{T} R}{\lambda} J^{*}(\mathrm{w})
$$

$w=\pi_{K}\left(R^{\mathrm{T}} \mathrm{g} / \lambda\right)$, that is $R^{T}(\mathrm{~g}-v)=\pi_{\lambda \mathrm{K}}\left(R^{\mathrm{T}} \mathrm{g}\right)$.Combined formula 7 has

$$
\lambda \partial \mathrm{J}\left(R^{-1} v\right)=\lambda \partial J(u)=\pi_{\lambda \mathrm{K}}\left(R^{\mathrm{T}} \mathrm{g}\right)
$$

The above formula shows that in convolution problems, the derivative of the regular term can be calculated by nonlinear projection $\pi_{\lambda \mathrm{K}}\left(\mathrm{R}^{\mathrm{T}} \mathrm{g}\right)$.

\section{B. RL_TV regularization algorithm based on Chambolle projection}

Through the above analysis, it is concluded that the canonical derivative of full difference can be calculated by chambolle projection $\pi_{\lambda \mathrm{K}}\left(\mathrm{R}^{\mathrm{T}} \mathrm{g}\right)$ in convolution problem. Based on this, it is obtained a new iterative formula of TV regularized $\mathrm{RL}$ algorithm. In RL algorithm with TV regular term, let the derivative of objective function be zero.

$$
\int h(-x) \mathrm{d} x-h(-x) * \frac{i(x)}{(h * o)(x)}-\lambda \nabla J_{T V}(o)=0
$$

It is further simplified as follows:

$$
h(-x) * \frac{i(x)}{(h * o)(x)}=1-\lambda \nabla J_{T V}(o)=1-\pi_{\lambda K}\left(R^{\mathrm{T}} i\right)
$$

The following iterative formula is obtained as:

$$
\begin{aligned}
o_{k+1}(x, y)= & \frac{o_{k}(x, y)}{1-\pi_{\lambda K}\left(R^{\mathrm{T}} o_{k}\right)}[h(-x,-y) \\
& \left.* \frac{i(x, y)}{\left(h * o_{k}\right)(x, y)}\right]
\end{aligned}
$$

This formula is the iterative formula of RL algorithm based on chambolle projection TV regularization. And it is applied to the reconstruction of MCS. TheShearlet coefficients of the original image $Y \in R^{N}$ can be represented as follows:

$$
Y=\alpha_{p_{0}}^{Y} \varphi_{p_{0}}+\sum_{p=p_{0}}^{P} \sum_{s} \hat{\theta}_{p, s}^{Y} \phi_{p, s}
$$

Gaussian random matrix is selected as sampling matrix, a nd TV regularization RL algorithm based on chambolle project ion is used to solve the following equation:

$$
\min \left\|\Psi_{w}^{T} \hat{\theta}_{p, s}^{(X)}\right\|_{T V} \text { s.t. } \quad y_{p, s}=\hat{\Phi}_{p, s} \Psi_{w}^{T} \theta_{p, s}^{(X)}
$$

In order to ensure $\Psi_{w}^{T} \hat{\theta}_{p, s}^{(X)}$ can accurantly reconstruct $y_{p, s}$ and make $\Psi_{w}^{T} \hat{\theta}_{p, s}^{(X)}$ sparse, the number of $m$ measurements should subject to $m \geq\left(C \cdot n \cdot K^{\prime}\right) \cdot \sqrt{\frac{N_{P}}{B}} \cdot\left(\log N_{p}\right)^{2}: N_{p}$ is the dimension of $p t h$ scale for original image.

Finally, The high resolution image reconstructed by MCS method is as follows:

$$
X=\alpha_{p_{0}}^{\left(X_{C S}\right)} \varphi_{p_{0}}+\sum_{p=p_{0}}^{P} \sum_{s} \hat{\theta}_{p, s}^{Y} \phi_{p, s}
$$

\section{EXPERIMENTAL RESULTS AND ANALYSIS}

In order to prove the effectiveness of this algorithm, The cameraman and Barbara images with Poisson noise are selected as test images, and the image size is $256 \times 256$. The total scale number of decomposition is $P$, and the number of directional subbands corresponding to each scale in high frequency region is $d_{p}=2^{p}+2$. In order to ensure the accurate reconstruction of subband information, the observation times of each direction subband is $m_{p, s}=C_{1} \cdot\left(N_{p} / d_{p}\right) \cdot \tau \cdot \log \left(N_{p}\right)$ where $\mathrm{C}$ is a constant, $N_{p}$ represented the dimension of $p$ th subband on the scale (the dimensions of subbands in different directions are consistent in the same scale). According to the scale decomposition characteristics of LP in DST, it can be seen that $N_{p}=N \cdot 2^{-2(P-p)}, N$ is the dimension of the original image. $\tau$ is the sparsity of the directional subband. The higher the scale, the higher the corresponding sparsity. If $\tau=C_{2} \cdot 10^{-(p+1)} \cdot s_{p}\left(C_{2}\right.$ is a constant $), P=3, C_{1}=4, C_{2}=2.5$ then the number of observations is shown in TABLE I.The total number $M=m_{0}+\sum_{p=1}^{P} \sum_{S} m_{p, s} \cdot s_{p}=14240 \quad$ of $\quad \mathrm{MCS}$ observations can be obtained from TABLE I. The results show that the convergence threshold is 0.005 , the regularization factor is 0.02 , the iteration times is set to 30 , and the number of internal chambolle iterations is $15, \tau=1 / 8$. Under the same 
conditions, the validity of the proposed model is verified by comparing with the standard full difference regularized RL iterative algorithm. The the recovery image quality is compared from the subjective visual effect and the objective index. The objective quantitative indexes are MSE, SNR and ISNR [20].

TABLE I. The value of $m_{p, s}$ with different scales

\begin{tabular}{|c|c|c|c|c|}
\hline & $N_{p}$ & $S_{p}$ & $\tau$ & $m, s$ \\
\hline$p=0$ & $32 * 32$ & 0 & 1 & $\begin{array}{r}1 \\
024\end{array}$ \\
\hline$p=1$ & $64 * 64$ & 4 & $2.5 \times 10^{-2} \times 4$ & $\begin{array}{c}1 \\
479\end{array}$ \\
\hline$p=2$ & $128 * 128$ & 6 & $2.5 \times 10^{-3} \times 6$ & $\begin{array}{c}6 \\
90\end{array}$ \\
\hline
\end{tabular}
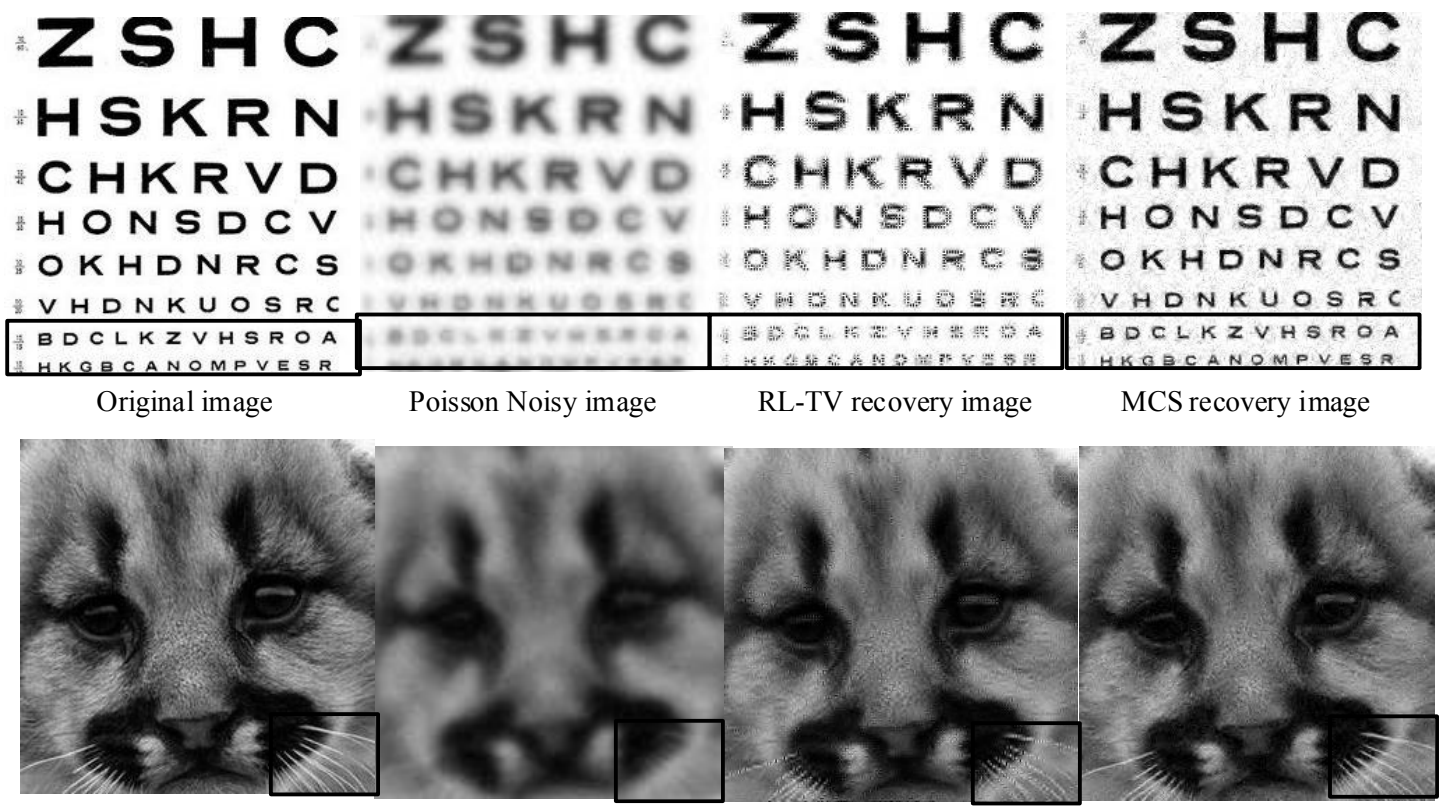

Original image

Poisson Noisy image

RL-TV recovery image

MCS recovery image

Fig. 2. The image reconstruction results of two methods

TABLE II The comparison of reconstruction results of two methods(The unit of SNR is dB)

\begin{tabular}{|c|c|c|c|c|}
\hline \multirow{2}{*}{ Image } & Index & $\begin{array}{c}\text { Poisson } \\
\text { noisy image }\end{array}$ & RL_TV & MCS \\
\hline \multirow{3}{*}{ Eyetest } & MSE & 1.19 & 654.971 & 588.179 \\
\cline { 2 - 5 } & SNR & 5.785 & 7.891 & 8.698 \\
\cline { 2 - 5 } & ISNR & 1.01 & 0.9639 & 0.8737 \\
\hline \multirow{3}{*}{ Raccoon } & MSE & 646.407 & 558.120 & 313.432 \\
\cline { 2 - 5 } & SNR & 7.112 & 7.809 & 10.311 \\
\cline { 2 - 5 } & ISNR & 1.02 & 0.8539 & 0.4761 \\
\hline
\end{tabular}

It can be seen from Fig. 2.that the image restored by the traditional RL_TV method is blurred seriously, and there is obvious confusion when reconstructing the edge, contour and other details of the image. As can be seen from Fig. 2., the area in the black box reflects the obvious difference between the traditional RL_TV and the restoration image details in this paper. When some details of the degraded image are seriously deficient, the image reconstructed by the traditional RL_TV method is not ideal. The MCS method can basically restore the fine details of the image. This is because MCS method changes the image signal into sparse signal by sparse representation, and CS theory is more suitable for 
reconstructing the signal with strong sparsity. It can be seen from the original image that there are slight noises and artifacts in the white smooth area of the image reconstructed by MCS. This is because MCS algorithm can effectively collect the information of high-frequency sub-band, and retain all the information of low-frequency sub-band, so that the details of the image remain intact. At the same time, due to the introduction of rl-tv algorithm, it can effectively remove the influence of Poisson noise and achieve good reconstruction effect.
As can be seen from TABLE II, the average SNR value of MCS method is $2.5 \mathrm{~dB}$ higher than that of RL_TV method, and the MSE average value is 176 lower; therefore, the algorithm in this paper is more effective for image reconstruction.

In this experiment, in order to compare the advantages of the MCS algorithm based on DST, we use the traditional CS algorithm and the Curvelet based MCS algorithm to reconstruct the image respectively. In order to better compare the reconstruction effect, the sampling rate is set as 30\%. Fig.3. gives the simulation results of two images.
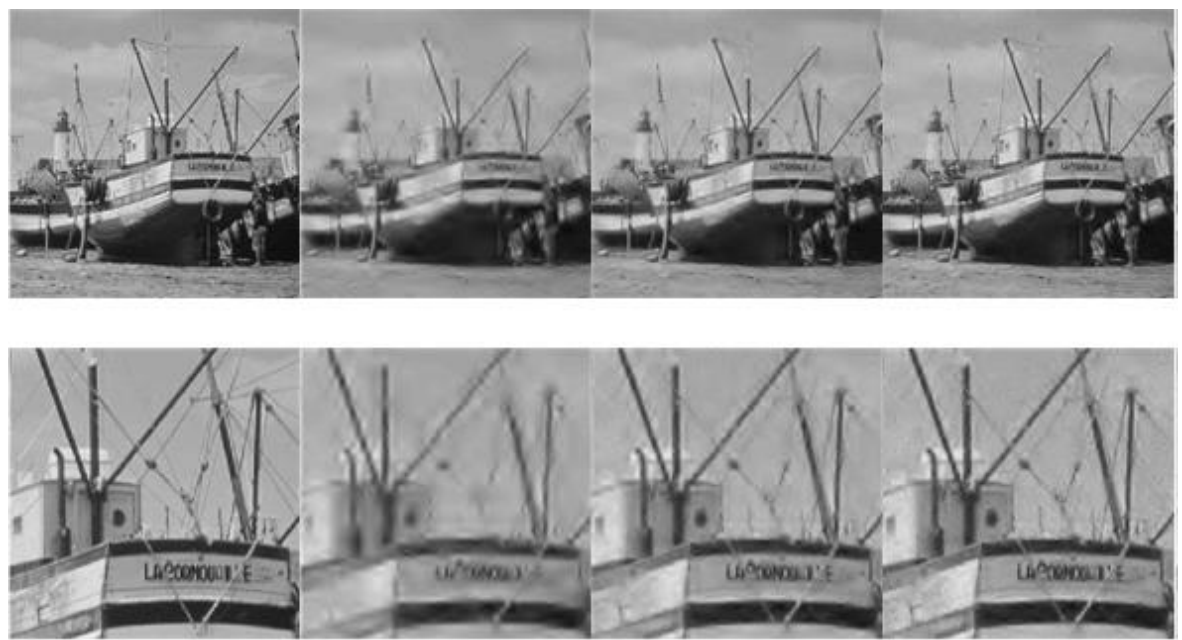

Fig.3. The reconstruction results of image Boats. From first to forth row: Boats image and its detail part; Reconstructed results using traditional CS, PSNR=22.12; Reconstructed results using Curvelet based MCS, PSNR=25.63; Reconstructed results using our scheme, PSNR=30.03.

It can be seen from Fig.3. that the traditional CS algorithm has great distortion, because the traditional algorithm does not retain the low-frequency information of the image, and there is a large amount of useless information due to non adaptive sampling. The reconstruction quality of MCS algorithm based on Curvelet is slightly better than that of traditional CS algorithm. However, due to the lack of FDFB in Curvelet, it is necessary to overcome the interference caused by spectrum aliasing, so the reconstruction effect is affected. This algorithm can effectively decompose the image with FDFB while retaining the low-frequency components, and sample the information on the high-frequency subband to the maximum extent, At the same sampling rate, the quality of the reconstructed image is obviously better than that of the contrast algorithm.

\section{CONCLUSIONS}

The most important feature of MCS method is established inShearlet domain. This algorithm can effectively reduce the sampling value and ensure the quality of the reconstructed image. By applying the successful application of nonlinear projection based on closed convex set in image denoising, the nonlinear projection equivalent to the total differential derivative in image deconvolution is derived. The nonlinear projection is used instead of the derivative of total difference to keep more details of image. In order to solve the problem of inaccuracy of reconstruction results caused by human loss of information in the process of derivation of regularization term, a new fully differential RL iterative algorithm based on nonlinear projection of closed convex sets is proposed. Based on this algorithm, the image reconstruction model and algorithm are given. Finally, the simulation results show the effectiveness of the new algorithm.

\section{REFERENCES}

[1] Dai J , François Goudail. Precision analysis of arbitrary full-Stokes polarimeters in the presence of additive and Poisson noise. Journal of the Optical Society of America A, 2019, 36(7), 1229-1236.

[2] Dai J, Goudail F, Boffety M, et al. Estimation precision of full polarimetric parameters in the presence of additive and Poisson noise. Optics Express, 2018, 26(26),34081.

[3] Richardson W H. Bayesian-Based Iterative Method of Image Restoration. Journal of the Optical Society of America. 1972, 62(1),55-59.

[4] Dey N , Laure Blanc-Féraud, Zerubia J , et al. A deconvolution method for confocal microscopy with total variation regularization, IEEE International Symposium on Biomedical Imaging, Nano to Macro. IEEE, 2004.

[5] Nowak R D, Kolaczyk E D. A statistical multiscale framework for Poisson inverse problems. IEEE Transactions on Information Theory, 2000, 46(5), 1811-1825

[6] Scipioni M , Santarelli M F , Giorgetti A, et al. Negative binomial maximum likelihood expectation maximization (NB-MLEM) algorithm for reconstruction of pre-corrected PET data. Computers in Biology and Medicine, 2019, 115,103481. 
[7] Donoho D L.Compressed sensing. IEEE Transactions on Information Theory, 2006, 52 (4), 1289-1306.

[8] Ede J M , Beanland R . Partial Scanning Transmission Electron Microscopy with Deep Learning. Scientific Reports, 2020, 10(1),8332.

[9] Lei J , Liu W , Liu S, et al. Multiscale reconstruction algorithm for compressed sensing. Isa Transactions, 2014, 53(4), 1152-1167.

[10] Qiao W , Yang Z . Forecast the electricity price of U.S. using a wavelet transform-based hybrid model. Energy, 2020,116704.

[11] Ahmed Y S , Arif A F M , Veldhuis S C . Application of the wavelet transform to acoustic emission signals for built-up edge monitoring in stainless steel machining. Measurement, 2020, 154,107478 .

[12] Fernandez L, Perez M , Orduna J M . Visualization of DNA methylation results through a GPU-based parallelization of the wavelet transform. Journal of supercomputing, 2019, 75(3), 1496-1509.

[13] Parvathy V S , Pothiraj S , Sampson J . A novel approach in multimodality medical image fusion using optimalShearlet and deep learning. International Journal of Imaging Systems and Technology, 2020.

[14] Ushio A , Yukawa M . Projection-Based Regularized Dual Averaging for Stochastic Optimization. IEEE Transactions on Signal Processing, 2019, (10),1-1.

[15] Kriel T L , Schweighofer M . On the Exactness of Lasserre Relaxations and Pure States Over Real Closed Fields. Foundations of Computational Mathematics, 2019 , 19(6), 1223-1263.

[16] Ren Z , Li Z . Imaging of elastic seismic data by least: quares reverse time migration with weighted L2- norm multiplicative and modified total ariation regularizations. Geophysical Prospecting, 2020, 68(2), 50-56.

[17] Cheng Z , Wang R . Deep stacking 11-norm center configuration convex hull and its application in fault diagnosis of rolling bearing. Mechanism and Machine Theory, 2020, 143,103648.

[18] Eldaly A K, Altmann Y, Perperidis A, et al. Deconvolution and Restoration of Optical Endomicroscopy Images. IEEE Transactions on Computational Imaging, 2019,194-205.

[19] Wu H , Li R , Kwok N M , et al. Restoration of low-informative image for robust debris shape measurement in on-line wear debris monitoring. Mechanical Systems and Signal Processing, 2019, 114(1),539-555.

[20] Kumari P, Sujediya G, Upadhyaya V . Variation Measurement of SNR and MSE for Musical Instruments Signal Compressed Using Compressive Sensing. Recent Trends in Communication and Intelligent Systems. 2020.

Pai Zhang (M'02-SM'06-F'09) received the B.S. and the M.S.degree in School of information engineering from Yanshan University, China, in 2006 and 2009 respectively, Ph.D. degrees in School of electrical engineering from the Yanshan University, Qinhuangdao, China, in 2013.From 2013 to now, she worked at College of Intelligence and Information Engineering Tangshan College, China,. Her current research interest is in the area of multiscale signal processing and applications in image analysis. Creative Commons Attribution License 4.0
(Attribution 4.0 International, CC BY 4.0)

This article is published under the terms of the Creative Commons Attribution License 4.0 https://creativecommons.org/licenses/by/4.0/deed.en_US 